\section{Fallstricke in Sport-Interventionsstudien}

\section{Kontrollierte Studien zeigen den Nutzen körperlicher Bewegung während und nach allogener Stammzelltransplantation (alloSCT). Doch bevor Empfeh- lungen an die Praxis gehen, sollten konfundierende Faktoren in Interven- tions- und Kontrollgruppe analysiert werden.}

örperliche Bewegung hilft gegen Belastungen wie Fatigue oder Funktionsverluste während und nach alloSCT, so das Ergebnis randomisierter Studien. Wichtig für die Interpretation der Ergebnisse sind Zusatzinformationen, z.B.

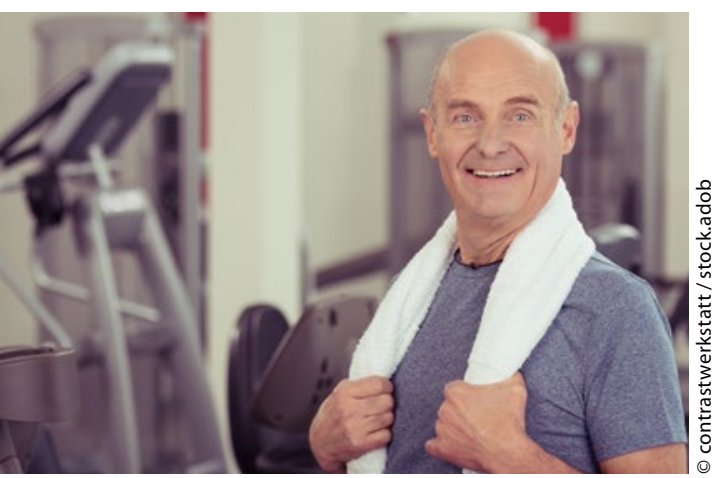

Körperlich aktiv auch nach allogener Stammzelltransplantation. über die Adhärenz der Teilnehmer in der Interventionsgruppe sowie über einen unkontrollierten, möglicherweise durch die Vergleichspatienten inspirierten Übungsbeginn auch in der Kontrollgruppe. Diesbezüglich wurden die Daten der noch laufenden PETRA-Studie analysiert. Darin hatte eine Subgruppe von 153 Patienten mit geplanter alloSCT über 1 Jahr entweder ein zunächst stationäres, dann ambulantes Ausdauer- und Krafttraining an 3-5 Tagen pro Woche oder Übungen zur progressiven Muskelrelaxation nach Jacobson durchgeführt. Die Adhärenz der Interventionsgruppe wurde anhand des Übungstagebuchs erfasst, kontaminierende Faktoren in der Kontrollgruppe per Fragebogen.

Die Adhärenz in der Interventionsgruppe variierte zwischen $66 \%$ während der stationären und $78 \%$ während der ambulanten Phase. Signifikant assoziiert war sie während der stationären Periode mit einer niedrigeren Fatigue-Rate $(\mathrm{p}=0,004)$. Die Adhärenz nach Entlassung korrelierte signifikant mit der Ausdauer $(p=0,003)$. Zudem war eine höhere Adhärenz nach 100 Tagen mit körperlicher Aktivität vor alloSCT und höherer Adhärenz nach Entlassung assoziiert $(\mathrm{p}=0,010$ und $\mathrm{p}=0,001)$. In der Kontrollgruppe gab es eine hohe Rate „kontaminierender" Aktivitäten: 54,2\% der Patienten waren 180 Tage nach Transplantation sportlich aktiv, 89,6\% gingen regelmäßig walken. Einflussfaktoren waren hier eine höhere isometrische Muskelkraft und Fatigue ( $\mathrm{p}=0,025$ und $\mathrm{p}=0,050$ ).

Fazit: In Studien, in denen der Einfluss körperlicher Übungsprogramme auf die Performance von Krebspatienten untersucht wird, sollten sorgfältig die Adhärenz in der Interventions- und mögliche körperliche Aktivitäten in der Kontrollgruppe dokumentiert werden.

Barbara Kreutzkamp

Kuehl R et al. Determinante of exercise adherence and contamination in a randomized controlled trial in cancer patients during and after allogenic HCT. Support Care Cancer. 2016; 24(10):4327-37.

\title{
4 Risikofaktoren für ZNS-Befall beim DLBCL
}

\begin{abstract}
Welche Patienten mit diffus-großzelligem B-Zell-Lymphom (DLBCL) haben ein erhöhtes Risiko für einen ZNS-Befall? In einer monozentrischen Studie kristallisierten sich 4 Risikofaktoren heraus, die eine ZNS-gerichtete Prophylaxe rechtfertigen.
\end{abstract}

D atie atienten mit DLBCL sind trotz hocheffektiver Chemoimmuntherapien langfristig von ZNS-Metastasen bedroht. Ob Rituximab dem vorbeugt, konnte bisher auch in Metaanalysen nicht geklärt werden. Präventive Therapien wie z.B. die Behandlung mit ZNSpenetrierenden hochdosierten Antimetaboliten sind denkbar, allerdings ist die Nutzen-Risiko-Abwägung schwierig. Um ein prognostisches Modell für das individuelle Risiko einer ZNS-Metasta- sierung beim DLBCL zu etablieren, werteten japanische Onkologen retrospektiv die Daten von 413 Patienten mit neu diagnostizierter Erkrankung aus, die zwischen 2004 und 2015 R-CHOP oder eine vergleichbare Therapie erhalten hatten.

Ein ZNS-Rückfall trat bei 27 Patienten auf (6,5\%). Das 5-Jahres-Risiko dafür errechnete sich mit $8,4 \%$, die mediane Zeit von Diagnosestellung bis zum ZNS-Progress mit 15 Monaten und das mediane Überleben nach Auftreten der ZNS-Metastasen mit 7 Monaten. In univariaten Analysen ergaben sich als Risikofaktoren für ZNS-Metastasen ein Ann-Arbor-Stadium 3/4, Albumin-Spiegel < 3,2 mg/l, mehr als ein extranodaler Herd sowie die Beteiligung retroperitonealer Lymphknoten. Einem prognostischen Modell mit diesen 4 Risikofaktoren zufolge ist die 5-Jahres-Wahrscheinlichkeit für eine
ZNS-Metastasierung bei Patienten, bei denen mindestens 3 dieser 4 Risikofaktoren vorliegen, signifikant höher als bei Patienten mit $\leq 2$ dieser Risikofaktoren (26,4 vs. $3,0 \%$; $\mathrm{p}<0,001)$. Mithilfe des Modells konnten die japanischen Wissenschaftler die Inzidenz und die Risikofaktoren für ZNS-Metastasen bei DLBCL-Patienten errechnen.

Fazit: Ein Ann-Arbor-Stadium von 3 oder 4, Albumin-Spiegel unter 3,2 mg/l, ein Befall retroperitonealer Lymphknoten sowie mehr als 1 extranodaler Befund eignen sich als Parameter für die RisikoStratifizierung im Hinblick auf eine ZNSMetastasierung, so das Ergebnis einer Berechnung anhand monozentrisch erhobener Daten.

Barbara Kreutzkamp

Kanemasa $Y$ et al. Central nervous system relapse in patients with diffuse large B cell lymphoma: analysis of the risk factors and proposal of a new prognostic model. Ann Hematol. 2016; 95(10):1661-9. 